\title{
Aspectos epidemiológicos e distribuição anatômica das diferentes formas clínicas do sarcoide equino no Rio Grande do Sul: 40 casos $^{1}$
}

\author{
Juliana S. Brum²*, Tatiana M. Souza ${ }^{3}$ e Claudio S.L. Barros ${ }^{4}$
}

\begin{abstract}
Brum J.S., Souza T.M. \& Barros C.S.L. 2010. [Epidemiological aspects and anatomic distribuition of equine sarcoid in the state of Rio Grande do Sul, Brazil: 40 cases.] Aspectos epidemiológicos e distribuição anatômica das diferentes formas clínicas do sarcoide equino no Rio Grande do Sul: 40 casos. Pesquisa Veterinária Brasileira 30(10):839-843. Programa de Pós-Graduação em Medicina Veterinária, Centro de Ciências Rurais, Universidade Federal de Santa Maria, Av. Roraima 1000, Camobi, Santa Maria, RS 97105-900, Brazil. E-mail: julianasbrum@yahoo.com.br

The files of histopathological exams carried out by the Laboratório de Patologia Veterinária of the Universidade Federal de Santa Maria (LPV-UFSM), between January 2000 and March 2010, were reviewed in search of cases of equine sarcoid. Forty cases were selected to determine epidemiological aspects and anatomical distribution of the various clinical forms of these neoplasms in horses from Rio Grande do Sul. Out of the cases in which the ages were registered in the histopathological reports, $73.0 \%$ (27/37) were 1 to 5 -year-old horses. Multiple sarcoids were observed in most of the affected horses (29/40 [72.5\%]). The fibroblastic form was most frequently observed and occurred in $42.2 \%(27 / 64)$ of the cases. In more than half of the cases (22/40 [55.0\%]), sarcoids had a multifocal distribution. Twenty two $(55 \%)$ out of the 40 horses evaluated had the tumors in the limbs.
\end{abstract}

INDEX TERMS: Diseases of skin, skin tumors, equine sarcoid, dermato-phatology, diseases of horses.

RESUMO.- Foram revisados todos os protocolos de exames histopatológicos, arquivados no Laboratório de Patologia Veterinária da Universidade Federal de Santa Maria (LPVUFSM), realizados entre janeiro de 2000 e março de 2010 , na busca de casos de sarcoide equino. Quarenta casos foram selecionados com o objetivo de determinar os aspectos epidemiológicos e a distribuição anatômica das diferentes formas clínicas deste tumor em equinos no Rio Grande do Sul. Dos casos que tiveram suas idades anotadas nos protocolos, $73,0 \%$ (27/37) eram de equinos entre 1 e 5 anos de idade. Múltiplos sarcoides foram observados na maioria

\footnotetext{
${ }^{1}$ Recebido em 21 de maio de 2010.

Aceito para publicação em 2 de julho de 2010.

2 Programa de Pós-Graduação em Medicina Veterinária, área de concentração em Patologia Veterinária, Centro de Ciências Rurais, Universidade Federal de Santa Maria (UFSM), Santa Maria, RS 97105900, Brasil. "Autor para correspondência: julianasbrum @ yahoo.com.br

${ }^{3}$ Pós-Doutorado, Laboratório de Patologia Veterinária, Departamento de Patologia, Centro de Ciências da Saúde, UFSM, Santa Maria, RS.

${ }^{4}$ Laboratório de Patologia Veterinária, Departamento de Patologia, Centro de Ciências da Saúde, UFSM, Santa Maria, RS.
}

dos equinos afetados (29/40 [72,5\%]). A forma clínica mais observada foi a fibroblástica, que ocorreu em 42,2\% (27/64) dos casos. Em mais da metade dos casos (22/40 [55,0\%]), os sarcoides tinham distribuição multifocal. Equinos que apresentavam tumores nos membros totalizaram 22 dos 40 (55\%) casos analisados.

TERMOS DE INDEXAÇÃO: Doenças de equinos, doenças de pele, tumores de pele, sarcoide equino, dermatopatologia.

\section{INTRODUÇÃO}

Entre todas as especialidades veterinárias, a dermatologia e a oncologia têm se destacado (Souza et al. 2006). Nos equinos, além de causar problemas econômicos, as lesões de pele são um importante problema estético. Depois do cão e do gato, o cavalo é o animal mais comumente atendido devido a problemas dermatológicos (Scott \& Miller Jr 2003). A pele é o local mais comum de neoplasmas nessa espécie, perfazendo aproximadamente $50 \%$ de todos os tumores. Entre os principais destacam-se sarcoide, carcinoma de células escamosas, papiloma e melanoma (Scott \& Miller Jr 2003). O sarcoide é considerado o tumor 
mais comum de pele dos equinos, com prevalência ao redor de 60\% (Rooney \& Robertson 1996).

Sarcoide é um tumor de pele localmente agressivo, porém não metastático, que afeta principalmente equídeos domésticos (Ginn et al. 2007). É descrito em qualquer raça, afeta indivíduos de todas as idades e ocorre em ambos os sexos (Genetzky et al. 1983). Acredita-se que a causa seja a infecção pelo papilomavírus bovino tipo 1 ou 2. As lesões frequentemente ocorrem em áreas de pele previamente traumatizadas, podendo se desenvolver em até seis meses pós-infecção (Scott \& Miller Jr 2003). Alguns sarcoides permanecem estáveis por toda a vida, enquanto outros crescem rapidamente, tanto em tamanho quanto em número (Bensignor et al. 2005). Ocasionalmente, esses tumores podem regredir espontaneamente (Scott \& Milller Jr 2003).

Devido a escassez de dados que caracterizam esse tumor na região de abrangência do Laboratório de Patologia Veterinária da Universidade Federal de Santa Maria (LPVUFSM), fez-se necessário determinar os aspectos epidemiológicos e a distribuição anatômica das diferentes formas clínicas do sarcoide no Rio Grande do Sul. Assim, pode-se auxiliar os clínicos e patologistas no diagnóstico dessa importante condição na medicina de equídeos.

\section{MATERIAL E MÉTODOS}

Foram revisados todos os protocolos de exames histopatológicos, arquivados no LPV-UFSM, realizados entre janeiro de 2000 e março de 2010, na busca de casos de sarcoide equino. Os protocolos referentes a esses casos foram separados e avaliados. Desses protocolos foram retiradas informações quanto à idade, ao sexo, à raça, à história clínica, à localização dos tumores e às descrições macroscópicas. As preparações histológicas de pele dos casos selecionados foram coradas pela hematoxilina e eosina e revisadas.

Os casos incluídos neste estudo foram selecionados por conterem o maior número das informações necessárias, possuírem preparações histológicas adequadas e serem provenientes do Rio Grande do Sul. Quanto ao sexo, os equinos foram divididos em machos e fêmeas, sem distinção quanto a castração. Foram ainda divididos em grupos etários previamente estabelecidos (Pierezan et al. 2009): menores de 1 ano, 1-5 anos, 6-14 anos e 15 anos ou mais. Em relação aos sítios anatômicos, os sarcoides foram considerados como únicos ou múltiplos e divididos em quatro grupos principais: cabeça, tron$\mathrm{co}$, membros e multifocais. Foram considerados multifocais aqueles casos em que havia lesões em pelo menos dois sítios anatômicos diferentes. Os sarcoides foram avaliados macro e histologicamente de acordo com critérios descritos na literatura (Martens et al. 2000) e divididos clinicamente em cinco categorias: verrucoso, fibroblástico, misto, oculto e nodular.

Macroscopicamente, os sarcoides incluídos como verrucosos eram bem circunscritos e tinham aspecto vegetante, com superfície irregular, contendo pequenas e numerosas projeções digitiformes. Os sarcoides incluídos como fibroblásticos tinham aparência carnosa e estavam, na maioria das vezes, ulcerados. Como nodular foram incluídos aqueles sarcoides que ocorriam como uma massa firme e recoberta por pele normal. Sarcoide oculto incluía todos os casos em que uma área alopécica e levemente elevada era posteriormente confirmada pela histologia. A expressão sarcoide misto foi utilizada para definir os casos em que mais de um desses padrões era observado em uma mesma massa.

Histologicamente, o critério utilizado para o diagnóstico incluía uma proliferação dérmica constituída por grande quantidade de células fusiformes na forma de ninhos ou feixes distribuídos em diferentes direções. Várias alterações epidérmicas eram observadas de acordo com cada tipo clínico de sarcoide e serviram para confirmar o padrão clínico. No tipo verrucoso, a hiperplasia epidérmica era caracterizada por projeções exofíticas (hiperplasia papilada) com acentuada hiperceratose. No tipo fibroblástico havia áreas ulceradas, por vezes intercaladas por áreas em que a epiderme estava levemente hiperplásica.

Desta maneira, foram selecionados 40 protocolos de exames histopatológicos de equinos que apresentavam sarcoide. Em 11 deles foram remetidos mais de um desses tumores, totalizando 64 massas cutâneas diferentes.

\section{RESULTADOS E DISCUSSÃO}

Dos 40 casos de sarcoide equino selecionados nos arquivos do LPV-UFSM, entre janeiro de 2000 e março de 2010, três não tinham o sexo informado. Dos 37 equinos que tinham o sexo descrito nos protocolos, $22(59,5 \%)$ eram fêmeas e 15 (40,5\%) eram machos.

A raça mais prevalente foi a Crioula $(77,5 \%$ dos casos), o que está de acordo com a realidade do estado do Rio Grande do Sul, onde esta é a principal raça utilizada em atividades esportivas e no manejo de bovinos em propriedades rurais (Pierezan 2009). Equinos Quarto de Milha (4/40), Puro Sangue Inglês (1/40), Pônei (1/40) e sem raça definida (3/40) totalizaram $22,5 \%$ dos casos.

A maior parte da literatura indica que o sarcoide não tem predileção por sexo ou raça, entretanto, alguns estudos relatam um aumento do risco nas raças de sela e uma diminuição da prevalência em raças de corrida (Scott \& Miller Jr 2003). Levando em consideração a etiologia desse tumor, a maior prevalência em cavalos de sela pode ser explicada pelo maior contato destas raças com bovinos em fazendas (Rooney \& Robertson 1996).

Do total de equinos, apenas três não tiveram sua idade anotada nos protocolos. Não se observaram equinos afetados com menos de um ano e nem com mais de 15 anos de idade. A maioria, 73,0\% (27/37), foi incluída entre um e cinco anos de idade. Os outros 10 cavalos $(27,0 \%)$ foram incluídos entre seis e 13 anos. Esse resultado guarda semeIhanças com outros estudos (Marti et al. 1993, Ginn et al. 2007) que descrevem que o sarcoide é mais observado em equinos entre três e seis anos, ou ainda com outros autores (Rooney \& Robertson 1996, Scott \& Miller Jr 2003) que mencionam que o tumor é comum antes dos sete anos e raro antes de um ano de idade.

Vários autores descrevem que as lesões são frequentemente múltiplas (Genetzky et al. 1983, Rooney \& Robertson 1996, Lloyd et al. 2003, Radostits et al. 2007). Dos 40 casos aqui relatados, $29(72,5 \%)$ apresentavam múltiplos sarcoides. Os outros 11 equinos $(27,5 \%)$ apresentavam um único tumor.

O sarcoide afeta qualquer parte do corpo, mas tem predileção pela cabeça, membros e abdômen ventral (White 
et al. 2002, Chambers et al. 2003, Lloyd et al. 2003). Neste estudo, em mais da metade dos casos (22/40[55,0\%]), os sarcoides apresentavam distribuição multifocal. Desses, em 72,7\% (16/22) havia tumor nos membros (Fig.1) e/ou no tronco (Fig.2) e em 12 casos (54,5\%) havia tumor na cabeça (Fig.3). No restante dos casos (18/40), observaram-se sete $(17,5 \%)$ apenas na cabeça, sete $(17,5 \%)$ somente nos membros e quatro $(10,0 \%)$ apenas no tronco.

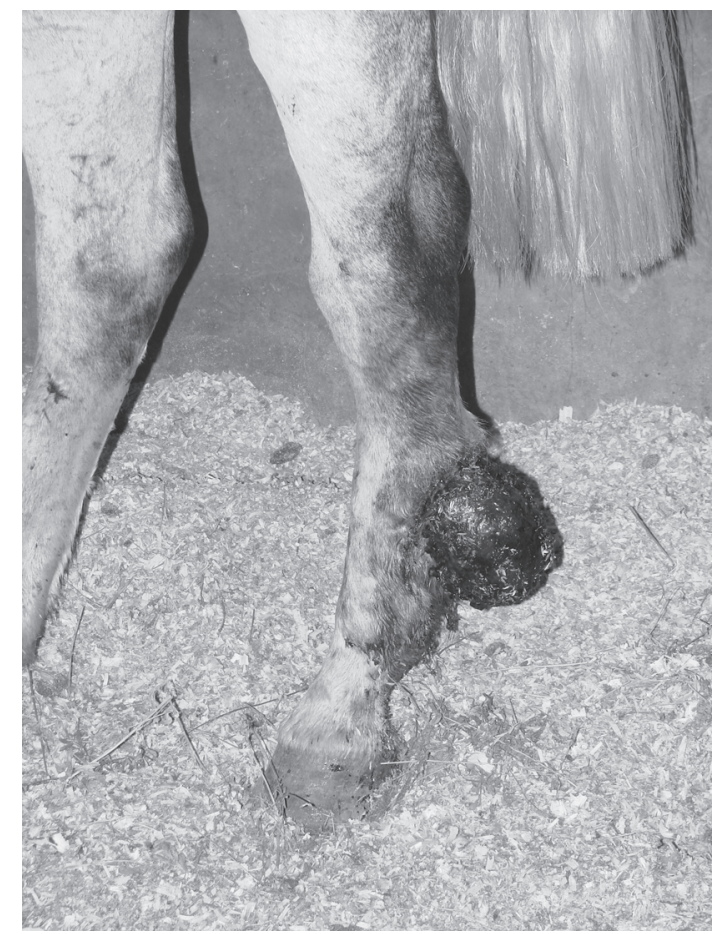

Fig.1. Sarcoide equino. Membro posterior. Massa ulcerada na região do calcâneo.

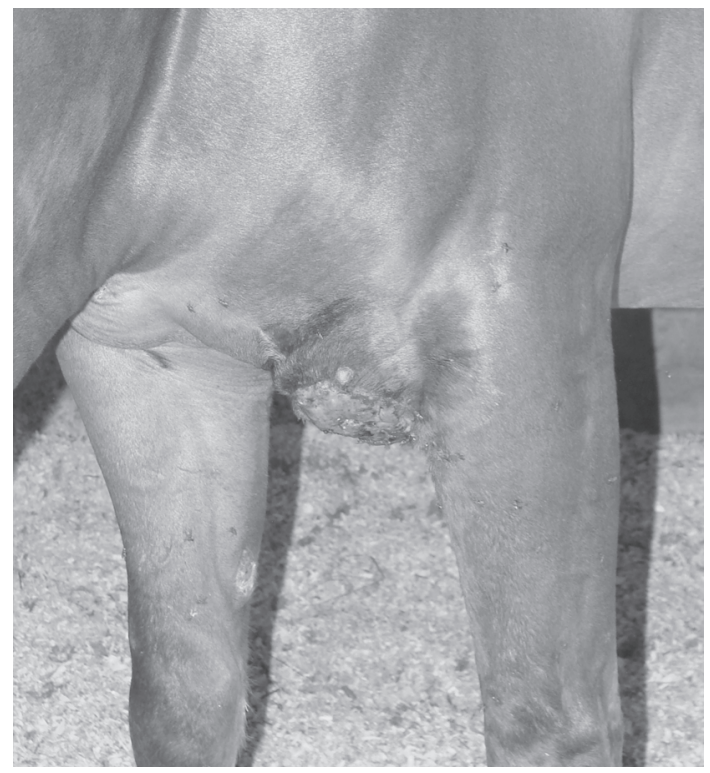

Fig.2. Sarcoide equino. Tronco. Massa infiltrativa e não delimitada na região peitoral.

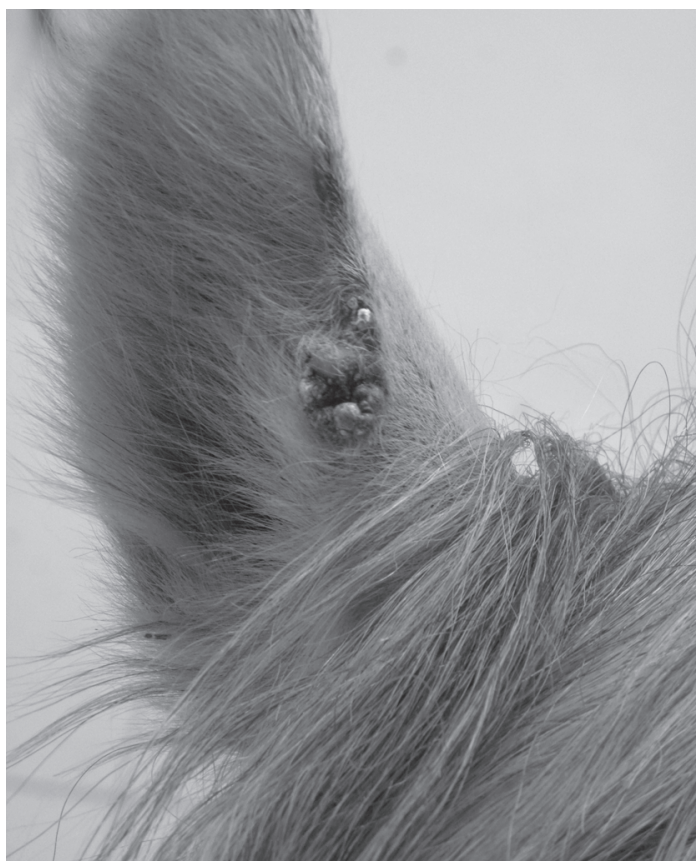

Fig.3. Sarcoide equino. Cabeça. Massa multilobulada, alopécica e de aspecto vegetativo no pavilhão auricular.

Equinos que apresentavam tumores nos membros totalizaram 22 dos 40 casos analisados.

De acordo com o aspecto clínico, os sarcoides são mais comumente divididos em quatro categorias: verrucoso, fibroblástico, misto e oculto (White et al. 2002, Scott \& Miller Jr 2003, Ginn et al. 2007, Radostits et al. 2007). Uma quinta categoria, a nodular, é ainda proposta (Martens et al. 2000). Diferentes formas podem ocorrer concomitantemente em um mesmo animal, em diferentes localizações ou em uma mesma lesão, dando um aspecto clínico variado (Bensignor et al. 2005), tanto quanto à forma como quanto ao tamanho (Rooney \& Robertson 1996, White et al. 2002, Scott \& Miller Jr 2003, Ginn et al. 2007). Das 64 massas cutâneas remetidas nos 40 casos aqui estudados, o tamanho descrito nos protocolos variou de 0,7 a 15 $\mathrm{cm}$. Sarcoides fibroblásticos podem crescer acentuadamente e lembrar um tecido de granulação exuberante. Infecções secundárias, principalmente miíase, podem ocorrer (Bensignor et al. 2005), como em três casos relatados neste estudo. A evolução clínica das lesões aqui estudadas variou de uma semana a três anos.

Os tipos clínicos de sarcoide estão diretamente relacionados com a histologia (Kottenbelt 2005). Assim, mediante a avaliação macroscópica e revisão histológica dos casos (Martens et al. 2000), a forma clínica mais observada foi a fibroblástica (Fig.4), ocorrendo em 42,2\% (27/64) dos tumores. O sarcoide misto (Fig.5), ao contrário do que relatam alguns autores (Lloyd et al. 2003), foi observado regularmente $(20 / 64[31,2 \%])$. A forma verrucosa, também ao contrário do que se acreditava, foi observada apenas em 17,2\% dos tumores (11/64). A forma oculta foi vista em cinco casos $(7,8 \%)$ e a nodular apenas em um caso $(1,6 \%)$. 


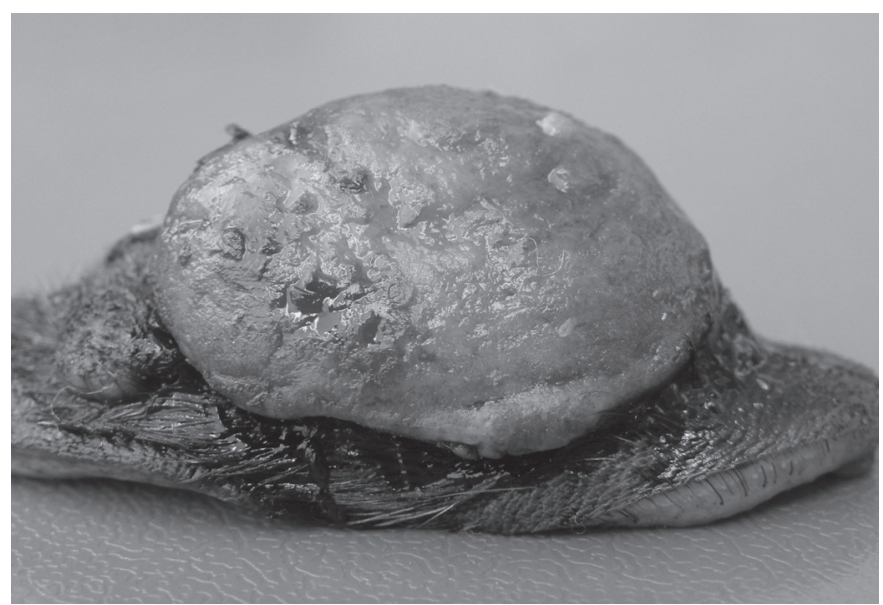

Fig.4. Sarcoide equino. Massa fibroblástica, difusamente ulcerada e com aparência carnosa.

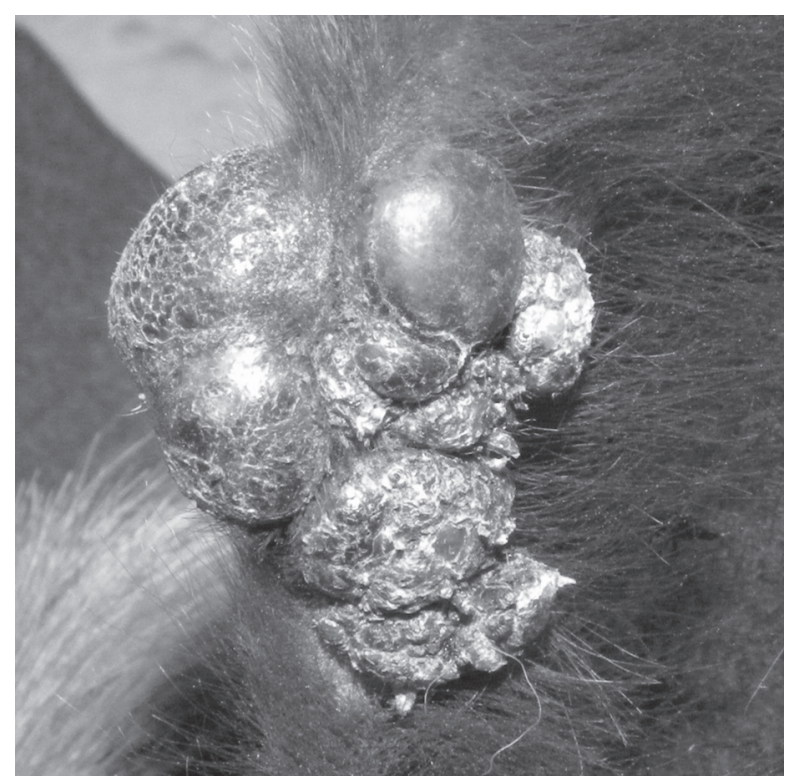

Fig.5. Sarcoide equino. Massa mista, constituída de componentes fibroblástico e verrucoso.

Acredita-se que a alta prevalência de sarcoides fibroblásticos e mistos observada neste estudo se deva ao fato que, com o tempo, as lesões podem mudar de padrão morfológico. Por exemplo, é descrito que os sarcoides verrucosos e ocultos, quando traumatizados, podem transformarse no tipo fibroblástico (Genetzky et al. 1983, Lloyd et al. 2003). Esses mesmos autores relatam também que sarcoides mistos são uma transição de verrucoso para o fibroblástico. Outra hipótese é que esta baixa prevalência da forma verrucosa pode ter relação com a região geográfica estudada. Sarcoides ocultos e verrucosos são incomuns na África e na Austrália, região situada na zona tropical sul, mas são particularmente comuns no Reino Unido, região subtropical norte (Scott \& Miller Jr. 2003).

Dos protocolos em que havia correlação das massas com os locais de origem, as formas fibroblástica e mista foram mais prevalentes nos membros (8/18 e $7 / 14$, res- pectivamente). A forma verrucosa foi mais prevalente na cabeça (3/4). Quando se compara esses resultados com os encontrados na literatura, pôde-se perceber que os achados são comuns. Alguns autores (Scott \& Miller Jr 2003, Salgado et al. 2008) afirmam que o tipo fibroblástico é mais comum nos membros e que o verrucoso é mais comum na cabeça. É importante lembrar que os membros são locais constantemente traumatizados podendo, assim, haver transformação entre os tipos de sarcoide, como mencionado anteriormente.

Os diagnósticos diferenciais dependem das várias formas morfológicas que a lesão pode ter. Por exemplo, do tipo verrucoso, inclui papiloma e carcinoma de células escamosas; e do tipo fibroblástico, tecido de granulação exuberante e habronemose (Scott \& Miller Jr 2003). Na nossa realidade a pitiose também deve ser colocada na lista de diagnósticos diferenciais devido à alta prevalência desta doença (Leal et al. 2001) e semelhança clínica que pode ter com o sarcoide fibroblástico.

\section{CONCLUSÕES}

Embora o sarcoide equino seja frequentemente visto na rotina da clínica e da patologia veterinária, são escassos os estudos disponíveis sobre este tumor. Com base nos achados deste estudo, pode-se concluir que os equinos afetados são principalmente fêmeas (relação macho:fêmea de 0,7), têm principalmente entre um e cinco anos de idade e pertencem a raça Crioula. Em relação à distribuição anatômica, tumores múltiplos são mais comuns (relação únicos:múltiplos de 0,4$)$. Os sítios anatômicos mais prevalentes quanto ao desenvolvimento do sarcoide são os membros e, independentemente da localização, a forma mais comum é a fibroblástica.

\section{REFERÊNCIAS}

Bensignor E., Groux D. \& Lebis C. 2005. As doenças de pele do cavalo. Organização Andrei Editora Ltda, São Paulo, p.84-87.

Chambers G., Ellsmore V.A., O'Brien P.M., Reid S.W.J., Love S., Campo M.S. \& Nasir L. 2003. Association of bovine papillomavirus with the equine sarcoid. J. General Virology 84:1055-1062.

Genetzky R.M., Biwer R.D. \& Myers R.K. 1983. Equine Sarcoids: Causes, diagnosis, and treatment. Compendium of Continuing Education for Practising Veterinarians 5:416-420.

Ginn P.E., Mansell J.E.K.L. \& Rakich P.M. 2007. Skin and appendages, p.553-781. In: Maxie M.G. (Ed.), Jubb, Kennedy and Palmer's Pathology of Domestic Animals. Vol.1. $5^{\text {th }}$ ed. Elsevier, Philadelphia.

Kottenbelt D.C. 2005. A suggested clinical classification for the equine sarcoid. Clinical Techniques in Equine Practice 4:278-295.

Leal A.T., Leal A.B.M., Flores E.F. \& Santurio J.M. 2001. Pitiose. Ciência Rural 31:735-743.

Lloyd D.H., Littlewood J.D., Craig J.M. \& Thomsett L.R. 2003. Practical Equine Dermatology. Blackwell Science Ltda, lowa, p.63-99.

Marti E., Lazary S., Antczak D.F. \& Gerber H. 1993. Report of the first international workshop on equine sarcoid. Equine Vet. J. 25:397407.

Martens A., De Moor A., Demeulemeester J. \& Ducatelle R. 2000. Histopathological characteristics of five types of equine sarcoid. Res. Vet. Sci. 69:295-300. 
Pierezan F. 2009. Prevalência das doenças de equinos no Rio Grande do Sul. Dissertação de Mestrado em Medicina Veterinária, Universidade Federal de Santa Maria, Santa Maria, RS. 163p.

Pierezan F., Rissi D.R., Rech R.R., Fighera R.A., Brum J.S. \& Barros C.S.L. 2009. Achados de necropsia relacionados com a morte de 335 eqüinos: 1968-2007. Pesq. Vet. Bras. 29:275-280.

Radostits O.M., Gay C.C., Hinchcliff K.W. \& Constable P.D. 2007. Veterinary Medicine: A textbook of the diseases of cattle, horses, sheep, pigs, and goats. $10^{\text {th }}$ ed. Saunders Elsevier, Philadelphia, p.1307-1438.

Rooney J.R. \& Robertson J.L. 1996. Equine Pathology. lowa State University Press, lowa, p.287-307.
Salgado B.S., Ferreira T.S., Viloria M.I.V. \& Conceição L.G. 2008. Avaliação clínica e epidemiológica dos casos de sarcoide equídeo atendidos no Hospital Veterinário da Universidade de Viçosa. Veterinária e Zootecnia 15(Supl.):6-8.

Scott D.W. \& Miller Jr W.H. 2003. Equine Dermatology. Saunders, Saint Louis, p.698-795.

Souza T.M., Fighera R.A., Irigoyen L.F. \& Barros C.S.L. 2006. Estudo retrospectivo de 761 tumores cutâneos em cães. Ciência Rural 36:555-560.

White S.D., Evans A.G. \& Van Metre D.C. 2002. Diseases of the skin, p.1200-1232. In: Smith B.P. (Ed.), Large Animal Internal Medicine. $3^{\text {rd }}$ ed. Mosby, Saint Louis. 